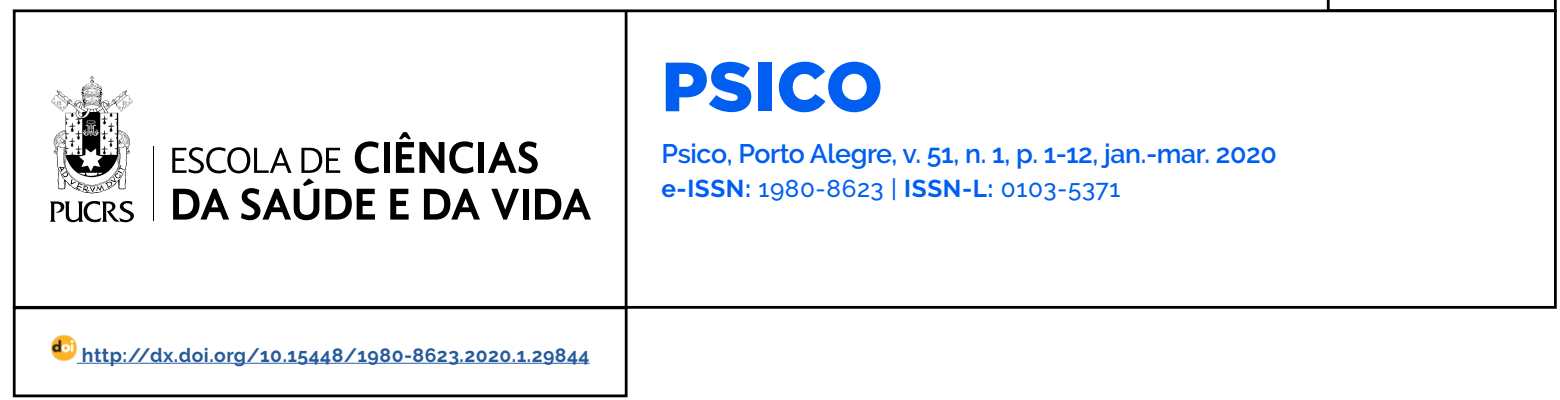

ARTIGOS

\title{
Familiaridade interfere no reconhecimento e na natureza da representação memorizada, não no processo de recuperação
}

\author{
Familiarity interferes in the recognition and nature of the memorized representation, not \\ in the process of retrieval \\ La familiaridad influye en el reconocimiento y en la naturaleza de la representación \\ memorizada, no en el proceso de recuperación
}

\author{
Isabella Wada e Pucci ${ }^{1}$ \\ orcid.org/0000-0002-0630-3999 \\ isabellawadap@gmail.com
}

\section{César Galera ${ }^{1}$}

orcid.org/0000-0002-3021-7126

algalera@usp.br

Recebido em: 20 fev. 2018

Aprovado em: 28 jun. 2019

Publicado em: $x x$ xxx, 2020.
Resumo: Nossa capacidade de armazenamento é maior para estímulos com os quais estamos mais familiarizados. No entanto, os processos especificos afetados pela familiaridade ainda são pouco conhecidos. Nós investigamos o efeito da familiaridade sobre a recuperação de representações visuais em uma tarefa de reconhecimento utilizando o paradigma de dicas retroativas. Uma tarefa de interferência visual irrelevante (Ruido Visual Dinâmico) foi utilizada como indicador da natureza visual das representações recuperadas. A familiaridade, definida como a exposição repetida ao mesmo conjunto de estímulos, foi manipulada entre dois grupos de participantes. Para um grupo (Grupo com Estímulos Repetidos) as provas experimentais continha estímulos sorteados de um mesmo conjunto de 8 (oito) caracteres chineses. Para o outro grupo (Grupo com Estímulos Inéditos) as provas eram formadas por estímulos inéditos, sorteados de um conjunto de 483 caracteres chineses. O RVD foi apresentado no intervalo entre a dica retroativa e o estímulo teste em metade das provas. $O$ desempenho de ambos os grupos na tarefa de reconhecimento melhora ao longo da sessão, mas de forma mais acentuada no grupo que trabalhou com Estímulos Repetidos. O efeito da dica se mantem inalterado ao longo da sessão e independe da familiaridade, entretanto a representação mais familiar mostra-se mais suscetivel à interferência do RVD, sugerindo que a exposição repetida aos mesmos estímulos permitiu a geração de representações visuais.

Palavras-chave: familiaridade, memória de trabalho, unitização, ruido visual dinâmico.

Abstract: Our ability to save is greater for more familiar stimuli than for less familiar. However, the specific processes affected by familiarity are still less known. We investigate the familiarity effect on the retrieval of visual representations in a recognition task, using the retroactive cue paradigm. A visual interference task (DVN-Dynamic Visual Noise) was used as visual nature indicator of recovered representations. The familiarity, defined as to the repeated exposure to the same stimuli set, was manipulated between two groups of participants. For one group (Group with Repeated Stimuli) the experimental tests contained stimuli drawn from the same set of 8 Chinese characters. For the other group (Group with Unpublished Stimuli) the tests were formed by unpublished stimuli, drawn from a set of 483 Chinese characters. DVN was presented in the interval between the retroactive tip and the test stimulus in half the tests. The performance of both groups improves on the task recognition throughout the experimental session, but more sharply at the group that worked with Repeated Stimuli. The retrieval effect remains unchanged throughout the session and independs of familiarity, however the more familiar representation is more susceptible to DVN, suggesting the repeated exposure to the same stimuli allowed the generation of visual representations.

Keywords: familiarity, working memory, unitization, dynamic visual noise.

Resumen: Nuestra capacidad de almacenamiento es mayor para estímulos familiares que para los estímulos poco familiares. Sin embargo, los procesos especificos afectados por la familiaridad son poco conocidos. Nosotros investigamos 
el efecto de la familiaridad sobre la recuperación de las representaciones visuales en una tarea de reconocimiento usando el paradigma de pistas retroactivas. Una tarea de interferencia visual irrelevante (Ruido Visual Dinámico) fue utilizada como indicador de la naturaleza visual de las representaciones recuperadas. La familiaridad, definida como la exposición repetida al mismo conjunto de estímulos, fue manipulada entre dos grupos de participantes. Para un grupo (Grupo con Estímulos Repetidos) las pruebas experimentales contenían estímulos sorteados de un mismo conjunto de 8 caracteres chinos. Para el otro grupo (Grupo con Estímulos Inéditos) las pruebas eran formadas por estímulos inéditos, sorteados de un conjunto de 483 caracteres chinos. El RVD fue presentado en el intervalo entre pista retroactiva y el estímulo test en la mitad de las pruebas. El desempeño de ambos los grupos en la terea de reconocimiento mejora a lo largo de la sesión experimental, pero mejora de forma más acentuada en el grupo que trabajó con los Estímulos Repetidos. El efecto de las pistas retroactivas se mantiene inalterado a lo largo de la sesión y no depende de la familiaridad, por otro lado, la representación más familiar se muestra más susceptible a la interferencia del RVD, sugiriendo que la exposición repetida a los mismos estimulos permitió la generación de representaciones visuales.

Palabras clave: familiaridad, memoria de trabajo, unificación, ruido visual dinámico.

A memória de trabalho é o sistema cognitivo responsável pela interface entre as exigências apresentadas por um ambiente complexo e o conhecimento armazenado na memória de longo prazo. É esse o sistema responsável pelo armazenamento temporário e pelo processamento da informação em tarefas cognitivas que exigem compreensão, aprendizagem e raciocínio (Baddeley, 2007). O sistema de memória de trabalho tem uma capacidade limitada tanto em termos do tempo em que uma determinada informação pode permanecer armazenada, quanto em termos da quantidade de informação que pode ser armazenada e/ou processada simultaneamente. Uma das estratégias utilizadas para se maximizar a capacidade de armazenamento e de processamento na memória de trabalho envolve a organização da informação em unidades mais complexas, em chunks (Miller. 1956). Um chunk é uma unidade de informações, associadas repetidamente ao longo do tempo e que funciona como uma representação coerente e integrada no momento da recuperação (Bower, 2000). O processo de formação de chunks, ou de unitização (unitization), permite que um número maior de objetos ou características seja agrupado e manipulado na memória de trabalho como uma representação única. A unitização, ou fusão, de um conjunto de objetos ou caracteristicas preexistentes em uma representação unitária possibilita a melhor utilização dos recursos disponiveis para a execução de uma tarefa em andamento (Kibbe \& Feigenson, 2016; Goldstone, 2000; McLaren \& Mackintosh, 2000). A utilização de estratégias de unitização pode melhorar o desempenho da memória e aumentar o benefício gerado pela repetição no processo de aprendizagem (Bader, Optiz, Reith, \& Mecklinger, 2014). A unitização também atenua os déficits associativos relacionados à idade (Ahmad, Fernandes, \& Hockley, 2015; D' Angelo et al., 2016), melhorando, por exemplo, a memória associativa em idosos (Memel \& Ryan, 2017).

Apesar de sua importância, os mecanismos subjacentes ao processo de unitização ainda são poucos conhecidos, principalmente quando se trata da Memória de Trabalho Visual. De acordo com Shiffrin e Lightfoot (1997) a unitização ocorre quando os recursos exigidos para execução de uma tarefa excedem a capacidade da memória de trabalho visual. McLaren e Mackintosh (2000) argumentam que o processo de unitização ocorre a partir do desenvolvimento de novas unidades perceptivas, através da exposição repetida aos componentes, que são agrupados em uma configuração específica, independente dos recursos exigidos pela tarefa. De acordo com esse modelo, as ligações entre diferentes elementos de um mesmo estímulo são fortalecidas quando ativadas concomitantemente em uma mesma representação. Com o tempo (repetição/treino) uma rede de conexões entre esses elementos será ativada simultaneamente, gerando uma representação unificada do estimulo visual. Desta forma, as características que são constantemente percebidas em determinada configuração, podem ser incorporadas em uma representação unitária, alterando qualitativamente o resultado do processamento dessa representação do estímulo (Schyns, Goldstone, \& Thibaut, 1998).

A apresentação repetida dos estímulos, ou de suas características especificas, geram novas associações entre esses componentes e, uma 
vez que as conexões foram criadas, a detecção de um componente do estímulo deve facilitar a detecção dos outros, através de uma ativação de propagação (spreading activation) (McClelland \& Rumelhart, 1985). Assim como a reativação de uma representação mental (através da repetição) reforça as relações entre os componentes preexistentes, uma vez que o processo gera novas, ou atualiza velhas ligações que nos auxiliam nos processos mnésicos (Vagnot, 2014).

A importância da coocorrência de características e das experiências anteriores com o objeto é evidenciada, por exemplo, no estudo de Goldstone (2000), no qual os participantes tinham que aprender a categorizar estímulos definidos por segmentos contínuos ou curvas. A categorização poderia ser realizada com base na apresentação de um único segmento ou com base em um conjunto de cinco segmentos. Tanto a acurácia como a análise do tempo de resposta indicam que os estímulos eram processados de forma mais eficiente quando apresentados em conjunto do que quando eram processados em segmentos isolados, reafirmando a importância da prática prolongada para a eficiência do desempenho e sugerindo que a detecção de um componente facilita a detecção dos outros componentes associados. James e Gauthier (2006) afirmam que a exposição repetida de estímulos ou objetos visuais geram mudanças tanto no comportamento, melhorando a acurácia e no tempo de resposta, como nos niveis de atividade cerebral quando os estímulos são reapresentados após curtos intervalos de tempo. Kandel, Schwartz, Jessell, Siegelbaum e Hudspeth (2014) ressaltam que a habilidade de determinar diferenças entre os estímulos visuais complexos é altamente sensivel às experiências anteriores do observador e que o aprendizado visual implícito, ou seja, a exposição repetida a determinados objetos complexos, produz mudanças na seletividade de respostas neuronais no córtex temporal inferior. Tais mudanças neuronais podem resultar tanto de discriminação ativa, quanto de visualização passiva, sendo muitas vezes manifestadas como um aperfeiçoamento da seletividade ao estímulo em vez de mudanças na frequência absoluta de disparo neural. Para Kandel et al. (2014) essa sintonia é precisamente o tipo de mudança neuronal que pode estar envolvida nas melhorias da discriminação perceptiva dos estímulos visuais. Sendo assim, o contato repetido com os estímulos, seria fundamental para o processo de unitização, uma vez que a exposição repetida aos estímulos proporciona uma codificação mais rápida se comparados a estímulos aos quais a pessoa nunca foi necessariamente exposta, ou aos quais foram menos expostas, influenciando diretamente a capacidade de memorização inclusive para itens simples armazenados na memória de trabalho (Brady, Störmer, \& Alvarez, 2016).

A unitização e a familiaridade são conceitos inter-relacionados. Delhaye e Bastin (2018) consideram que a representação criada a partir do processo de unitização torna as informações mais familiares do que quando consideradas individualmente (não unitizadas), tornando-as, dessa forma, mais facilmente recuperáveis. A familiaridade com o estímulo é uma forma indireta de avaliar a associação entre componentes que ocorre na unitização, uma vez que quanto menor o número de chunks utilizados na representação unitizada, maior a familiaridade com o estímulo (Perlman, Pothos, Edwards, \& Tzelgov, 2010). Para Parks e Yonelinas (2015) a unitização reflete uma forma especializada de aprendizagem associativa, onde a familiaridade com os estímulos influencia as condições as quais ocorre a associação semântica entre os componentes dos estímulos. A importância do componente semântico é evidenciada, por exemplo, por Orme, Brown e Riby (2017) que mostram que matrizes visuais aleatórias com alto conteúdo semântico são mais facilmente e melhor recordadas do que estímulos com baixo conteúdo semântico. Riby e Orme (2013) identificaram padrões eletrofisiológicos diferentes para matrizes com conteúdos semânticos altos e baixos, sugerindo que o conteúdo semântico pode reduzir a carga do processamento visual inicial (early visual processing) da informação visual, assim como dos processamentos mais tardios, associados à carga da memória, através da unitização de padrões complexos em formas familiares. Outros estudos corroboram com a 
suposição de que a familiaridade interfere nas estratégias iniciais do processamento, por exemplo, Reder e colaboradores (Reder, Paynter, Diana, Ngiam, \& Dickison, 2007; Reder, Liu, Keinath, \& Popov, 2016) sugerem que a familiaridade interfere no processo de codificação. A codificação de estímulos familiares exigiria menos recursos de memória de trabalho do que a codificação de estímulos pouco familiares.

Embora a importância da familiaridade sobre o desempenho em tarefas de memória em geral, e sobre os processos de codificação em particular, tenha sido bastante explorada, pouco ainda se sabe sobre os efeitos da familiaridade sobre os processos de recuperação da informação armazenada na memória de trabalho visual. Neste sentido, o desenvolvimento recente do paradigma de dicas retroativas (Griffin \& Nobre, 2003: Landman, Spekreijse \& Lamme, 2003) abre novas perspectivas para o estudo dos fatores que afetam o processo de recuperação da informação memorizada. Nesta tarefa, o participante memoriza uma cena visual com estímulos distribuídos no espaço. Posteriormente, depois que esses estimulos não estão mais disponiveis à percepção, o participante é informado, por uma dica espacial retroativa, sobre a localização do estimulo que terá a maior probabilidade de ser apresentado como estimulo teste. Os resultados mostram que o desempenho é melhor nas provas com dicas retroativas informativas, que indicam o local previamente ocupado pelo estimulo teste, do que nas provas com dicas neutras, onde nenhum localé indicado. Lepsien e Nobre (2006) sugerem que a atenção pode ser deslocada de forma dinâmica para localizações especificas na representação memorizada, permitindo que a informação relacionada à localização indicada, armazenada na memória de trabalho, seja recuperada e mantida em um estado ativo até que o estimulo teste seja apresentado e uma decisão possa ser tomada. A ativação da representação é subjacente ao processo de recuperação, entretanto a explicação para os beneficios gerados pela dica retroativa informativa (em tarefas de memória visual) ainda estão sendo investigados. Souza e Oberauer (2016) também consideram a recuperação como uma das hipóteses que explica o beneficio proporcionado pela dica retroativa. De acordo com esses autores, nas provas com dicas retroativas informativas o processo de recuperação, entendido como um acúmulo de informação necessária para a decisão, acontece de forma mais eficiente, ou mais rápida, do que nas provas sem as dicas retroativas, nas quais a tomada de decisão seria mais demorada pois seria desencadeada pela apresentação do estímulo teste.

Nós consideramos que o processo de recuperação da informação da memória de trabalho visual para um estado ativo se dá em termos da ativação de áreas corticais envolvidas também na percepção, em um processo equivalente à ativação das áreas corticais visuais nos processos de imaginação e percepção visual, tal como sugerido por Kosslyn (1994; Kosslyn, Thompson \& Ganis, 2006). Sendo assim, pode-se supor que essas representações recuperadas da memória de trabalho visual, também serão sensiveis à interferência de uma informação visual irrelevante, que atue sobre as mesmas áreas corticais ativas durante o processo de recuperação. Por exemplo, Quinn \& McConnell (1996) mostraram que apresentação de um Ruído Visual Dinâmico (RVD), semelhante ao chuvisco de uma tela de TV fora de sintonia, prejudica o desempenho em tarefas de imaginação, se o RVD é apresentado no período de codificação dos estímulos, ou no momento que esses estão sendo recuperados, ou seja, quando se encontram em um estado ativo, disponiveis à inspeção consciente como informação visual irrelevante. Evidências mais recentes sugerem que o RVD atua também durante o processo de recuperação da informação armazenada na memória de trabalho visual (Galera \& Quinn, 2014; Pucci \& Galera, 2014). Desta forma, neste estudo utilizamos o RVD para avaliar a natureza da representação recuperada da memória de trabalho visual. Nossa hipótese foi que a interferência do RVD seria tanto maior quanto menor a familiaridade do participante com o estímulo recuperado, seja porque a familiaridade permitiria uma recuperação mais rápida ou a representação familiar seria mais unitizada e estável.

No presente estudo investigamos o efeito da familiaridade com estímulos visuais no processo de recuperação de representações na memória 
de trabalho visual. A familiaridade com o estímulo foi investigada em uma tarefa de reconhecimento na qual um grupo de participantes foi exposto um pequeno conjunto de oito estímulos (Grupo com Estímulos Repetidos), enquanto os participantes de outro grupo foram expostos, a cada prova, a um novo conjunto de estímulos (Grupo com Estímulos Inéditos). Evidentemente a familiaridade resulta da interação dos participantes com os estímulos e deve ser estabelecida, ao longo da sessão experimental, em um processo equivalente à aprendizagem hebbiana (Hebb, 1961). Dessa forma, a repetição dos estímulos ao longo das provas deve ser considerada um fator que contribui para o estabelecimento da familiaridade. Nós consideramos que a repetição de um pequeno conjunto de oito estímulos durante toda a sessão experimental tornaria esses estímulos mais familiares aos participantes. Nossa suposição era que a repetição de estímulos visuais contribuiria para a formação de uma representação mental mais familiar, e, portanto, mais unitizada, para os estímulos apresentados repetidamente do que para estímulos inéditos (cf. Parks \& Yonelinas, 2015; Li, Mao, Wang \& Guo, 2017). Além disso, consideramos que esse processo tornaria a representação do estímulo mais unitizada e menos sensivel a interferência de informações visuais irrelevantes do que as representações para estímulos inéditos, não unitizados e com baixa familiaridade.

\section{Método}

\section{Participantes}

O experimento contou com 32 participantes voluntários, entre 18 e 37 anos ( $M=24,65 ; D P=1,14)$, de ambos os sexos (14 homens e 18 mulheres), com acuidade visual ou visão corrigida, que foram distribuidos em dois grupos experimentais. Todos os participantes assinaram o Termo de Consentimento Livre e Esclarecido e o projeto foi aprovado pelo Comitê de Ética da instituição.

\section{Materiais e estímulos}

Foram utilizados como estímulos 483 caracteres chineses, retirados do banco de dados de caracteres chineses Research Centre for the Humanities Computing da Universidade de Hong Kong (http://humanum.arts.cuhk.edu. hk/Lexis/Lexi-can recuperado em 14 de julho de 2015). Tendo em vista que o objetivo do presente estudo foi investigar a recuperação da informação na memória de trabalho visual, a utilização dos caracteres chineses como estímulos se deve ao fato de serem estímulos visuais ricos em traços, de dificil nomeação, o que contribuiria para que o participante não utilizasse estratégias verbais de memorização, e de difícil associação com a memória de longo prazo, considerando o contexto cultural ao qual o estudo foi dirigido.

Os estímulos foram apresentados em um monitor de LED de 21,5" (resolução $1024 \times 768$ pixels) e 60 Hz. A apresentação dos estímulos, assim como o registro das respostas foi realizada pelo utilitário E-Prime (Schneider, Eschman, \& Zuccolotto, 2002). Os estímulos, com aproximadamente 2 $\mathrm{cm}^{2}$ de área, foram apresentados nos vértices de um triângulo equilátero, equidistantes $2 \mathrm{~cm}$ do ponto de fixação central. O ponto de fixação foi uma moldura triangular com laterais de $0,5 \mathrm{~cm}$ e contorno desenhado com $1 \mathrm{~mm}$ de espessura. As dicas informativas e neutras e tiveram as mesmas dimensões que o ponto de fixação, mas com dois lados desenhados com $2 \mathrm{~mm}$ de espessura, apontando para uma das três posições que eram ocupadas pelos estímulos (superior, inferior direita e inferior esquerda). Na dica neutra os três lados do triângulo tiveram $2 \mathrm{~mm}$ de espessura. O estímulo teste teve as mesmas dimensões dos estímulos memorizados e foi apresentado no centro da tela. O RVD foi formado por uma matriz de $80 \mathrm{x}$ 80 pontos, cada ponto com $4 \times 4$ pixels, cobrindo uma área de aproximadamente $13,5 \times 13,5 \mathrm{~cm}$ no centro da tela do monitor. Metade dos pontos do RVD eram brancos e a outra metade os pontos eram pretos, sendo que a cada segundo, 50\% dos pontos tomados aleatoriamente, mudavam de preto para branco e vice-versa.

\section{Procedimento}

A Figura 1 apresenta de maneira esquemática a sequência de eventos em cada prova. A tarefa experimental consistia em uma tarefa de 
reconhecimento com dicas retroativas (Lepsien \& Nobre, 2006). Em cada prova o participante era instruido a memorizar um conjunto de três caracteres chineses, ao final do intervalo de retenção, uma dica retroativa espacial poderia informar qual seria o estímulo relevante para a tarefa de reconhecimento. O estímulo teste era apresentado depois da dica e o participante deveria responder se este era igual a um dos estímulos memorizados, ou não. Cada prova tinha início com a apresentação do ponto de fixação (1000 ms). Os três caracteres a serem memorizadas eram apresentados por 1500 ms. A dica retroativa, com duração de 250 ms, substituía o ponto de fixação $2500 \mathrm{~ms}$ depois da apresentação dos estímulos. O estímulo teste era apresentado 700 ms depois da dica e permanecia na tela até que o participante efetuasse a sua resposta. A resposta positiva (sim, o estímulo teste estava presente na cena inicial era dada pressionando-se o botão direito do mouse, e a resposta negativa (não, o estímulo teste não estava presente na cena inicial) pressionando o botão esquerdo do mouse. Um sinal de soma azul sinalizava uma resposta correta; e um sinal vermelho sinalizava uma resposta incorreta.

Em metade das provas o estímulo teste era igual a um dos três estímulos memórizados (provas positivas), e na outra metade era um estímulo novo (provas negativas). Em 75\% das provas a dica era informativa (apontava para o local em que o estímulo relevante para a tarefa havia sido apresentado), nas provas restantes a dica era neutra (apontava simultaneamente para as localizações de todos os estímulos). Em 50\% das provas o RVD era apresentado durante o intervalo de $700 \mathrm{~ms}$, entre a apresentação da dica e do estímulo teste (IDE), nas provas restantes a tela permanecia em branco. Durante a sessão experimental foram realizadas 138 provas, sendo que as 12 provas iniciais foram consideradas como treino. Um dos grupos de participantes trabalhou durante toda a sessão experimental com um conjunto de oito caracteres chineses (Grupo com Estímulos Repetidos), o outro grupo (Grupo com Estímulos Inéditos) trabalhou em cada prova com caracteres novos, retirados sem reposição do conjunto de 483 caracteres.

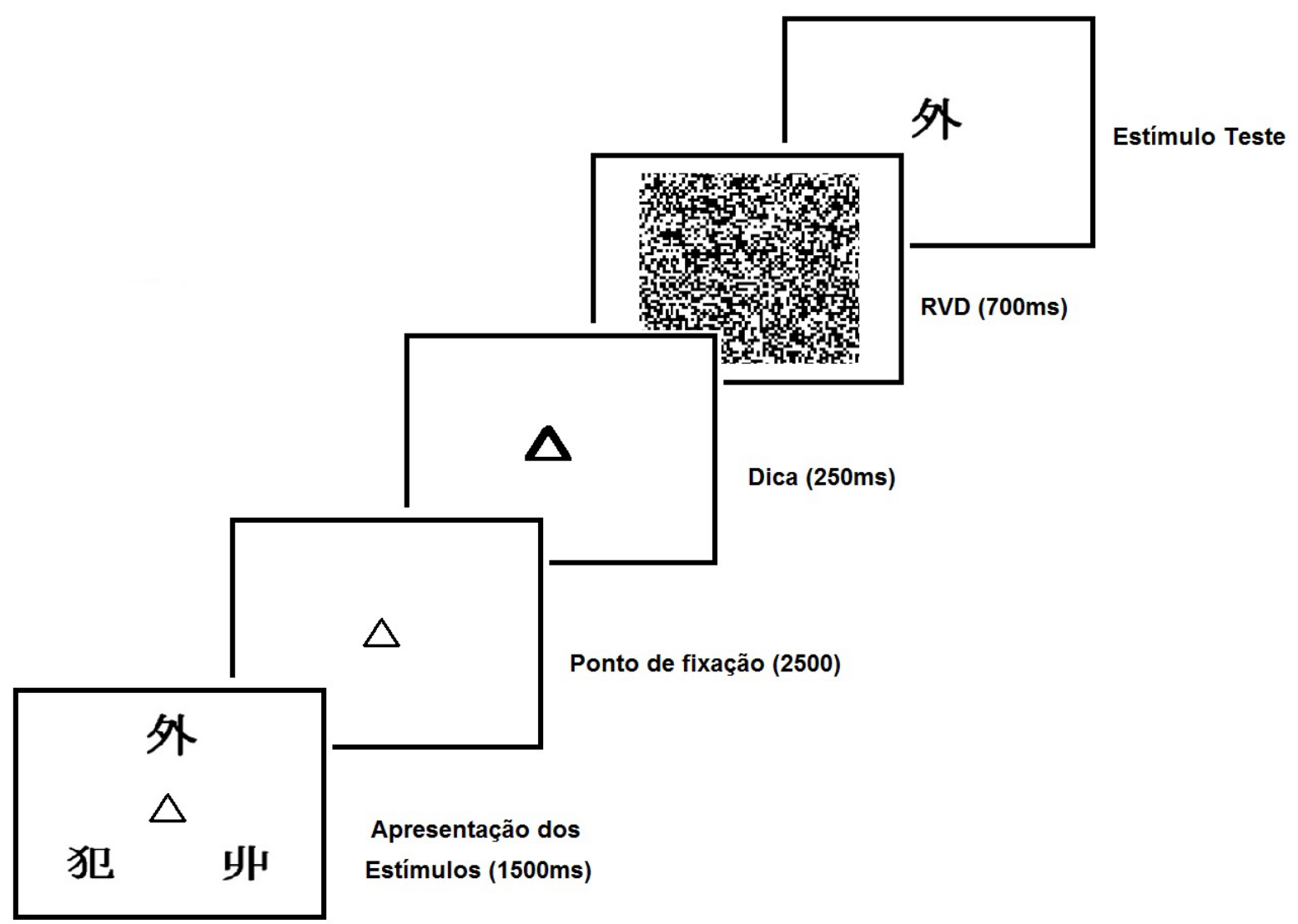

Figura 1 - Sequência de eventos de uma prova com dica informativa e apresentação do RVD. 


\section{Delineamento}

A familiaridade com os estímulos visuais (repetidos e inéditos) foi manipulada entre grupos de participantes. Em cada grupo, o tipo de dica (informativa e neutra), o tipo de prova (positiva, negativa) e a presença do RVD (ausente, presente) foram manipulados entre as provas. A sessão experimental foi dividida em três etapas consecutivas (Blocos de provas), cada uma formando um bloco de 42 provas.

\section{Resultados}

Os resultados da tarefa de reconhecimento foram analisados em termos do indice de discriminação $d^{\prime}$ (Snodgrass \& Corwin, 1988), e em termos da média dos tempos de resposta (TR) em milissegundos (ms). A média dos TRs foi estimada com base no tempo de resposta das respostas corretas, excluídos os valores abaixo de $300 \mathrm{~ms}$ e acima de $5000 \mathrm{~ms}$. Esses dados foram submetidos a análises de variância de modelo misto que levou em conta a familiaridade com os estímulos (alta - estímulos repetidos e baixa - estímulos inéditos), manipulada entre os dois grupos de participantes, e medidas repetidas nos tratamentos resultantes da combinação do tipo da dica (informativa, neutra), da presença do RVD (presente, ausente) e do bloco de provas (b1, b2 e b3). Quando necessário, estimamos para cada participante a taxa media de variação do desempenho ao logo dos blocos de provas através do coeficiente angular de uma regressão linear simples do desempenho em função do bloco de provas. O teste de Bonferroni foi utilizado para análises post-hoc.

A análise de variância confirma que $\mathrm{o}$ indice de discriminação é maior nas provas com dicas informativas $\left(d^{\prime}=1,57\right.$; $\left.E P M=0,35\right)$ do que nas provas com dicas neutras $\left(d^{\prime}=0,84 ; E P M=0,22\right)(F$ $\left.(1,30)=59,40, p<0,001, n_{p}{ }^{2}=0,66\right)$. O tipo de dica não interage de forma simples com nenhum dos outros fatores experimentais. A única interação significativa da qual esse fator participa é na interação quadrupla, com todos os outros fatores, da qual trataremos abaixo.

O desempenho melhora de forma significativa ao longo dos blocos de provas $(F(2,60)=9,26, p<$ $\left.0,001, n_{p}{ }^{2}=0,24\right)$ e essa melhora no desempenho ao longo dos blocos é mais acentuada para o grupo que foi exposto aos estímulos repetidos do que para os grupos que foi exposto a estímulos inéditos $\left(F(2,60)=3.39, p=0,04 ; n_{p}{ }^{2}=0,10\right)$. Para o grupo que trabalhou com estímulos repetidos o indice de discriminação aumenta em uma taxa de 0,29 do primeiro ao último bloco, enquanto para - grupo que trabalhou com estimulos inéditos essa taxa é de 0,10. É importante observar que a melhora no desempenho só acontece do segundo para o terceiro bloco de provas $(p=0,05)$ para o grupo exposto aos estímulos repetidos; já a variação do desempenho do grupo exposto aos estímulos inéditos não é significativa.

O efeito principal do RVD não chega a ser significativo $(p=0,25)$, mas esse fator tem efeitos diferentes nos dois grupos, como mostra a interação entre a presença do RVD e a familiaridade $\left(F(1,30)=5,79, p=0,02, n_{p}{ }^{2}=0,16\right)$. De acordo com essa interação, o desempenho do grupo que foi exposto a estímulos repetidos é pior na presença do RVD ( $\left.d^{\prime}=1,14 ; E P M=0,44\right)$ do que nas provas controle $\left(d^{\prime}=1,39 ; E P M=0,35\right)$ $(p=0,04)$. Já o desempenho do grupo exposto aos estímulos inéditos nas provas com RVD ( $d$ ' $=1,19 ; E P M=0,44)$ não pode ser considerado diferente do desempenho nas provas controle (RVD $\left(d^{\prime}=1,10 ; E P M=0,35\right)(p=1,0)$. Essa interação entre a presença do RVD e o tipo de estímulo (inédito e repetido) varia ao longo da sessão, com é evidenciado pela interação entre esses fatores e o bloco de provas $(F(2,60)=3.32, p=$ $0,04, n_{p}{ }^{2}=0,10$ ). De acordo com essa interação, o prejuizo médio causado pelo RVD no índice de discriminação nos dois primeiros blocos de provas do grupo de estímulo repetidos, de 0,33 $(p=0,03)$, tende a diminuir para $-0,01(p=0,97)$ no terceiro bloco. 
2.A

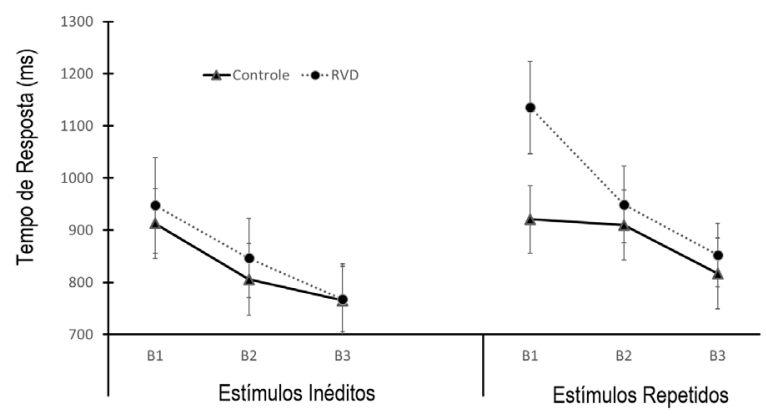

2.B

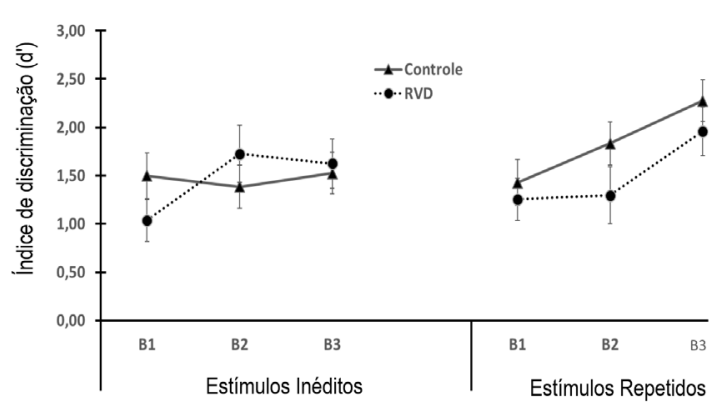

Figura 2 - Médias dos tempos de resposta (2.A) e dos índices de discriminação d' (2.B), obtidos em função do tipo de estímulo e da presença do RVD nos três blocos de provas da sessão experimental. Barras verticais indicam o erro padrão da média.

O tipo de dica também contribui com a variação do desempenho na interação quadrupla com os outros fatores manipulados $(F(2,60)=3,87, p$ $=0,03, n_{p}^{2}=0,11$ ). Para a análise dessa interação realizamos análises de variância separadas para os dados obtidos nas provas com dicas informativas e dicas neutras considerando a familiaridade com os estímulos como o fator manipulado entre grupos e as medidas repetidas nos fatores RVD e o bloco de provas. De acordo com a análise dos dados obtidos com a dica neutra nenhum dos fatores manipulados têm efeitos principais significativos sobre o desempenho (todos os ps $>0,02$ ). Nas provas com dicas informativas (Figura 2A) o desempenho é mais prejudicado pela presença do RVD ( $\left.d^{\prime}=1,5 ; E P M=0,27\right)$ do que nas provas sem o RVD $\left(d^{\prime}=1,7 ; E P M=0,24\right)$ $\left(F(1,30)=6,53, p=0,02, n_{p}^{2}=0,18\right)$. Esse efeito é mais acentuado para o grupo que trabalhou com estímulos mais familiares $(F(1,30)=7,10, p=0,01$, $\left.n_{p}{ }^{2}=0,19\right)$. Essa interação, avaliada pelo teste post hoc de Bonferroni, mostra que o desempenho nas provas com estímulos mais familiares é mais prejudicado pela presença do RVD $\left(d^{\prime}=1,5 ; E P M=\right.$ 0,38 ) do que nas provas sem o RVD ( $d^{\prime}=1,8 ; E P M$ $=0,34)(p=0,008)$. Já nas provas com estímulos menos familiares, o desempenho é o mesmo nas provas com e sem RVD ( $\left.d^{\prime}=1,5 ; p=0,99\right)$. $O$ desempenho também melhora de forma linear, a uma taxa de $0,3(r x y=0,99)$ do primeiro ao último bloco de provas $(F(2,30)=12,15, p<0,001$; $\left.n_{p}{ }^{2}=0,29\right)$. Essa taxa também varia em função da familiaridade $\left(F(2,60)=3,55, \mathrm{p}=0,03 ; n_{p}{ }^{2}=0,10\right)$. A taxa estimada para os estímulos repetidos é maior $(0,4)$ do que para estímulos inéditos $(0,2)$.

O desempenho também foi influenciado de forma significativa pela interação entre os três fatores analisados $\left(F(2,60)=3,5, p=0,04 ; n_{p}{ }^{2}=\right.$ 0,11 ). Essa interação mostra que tanto o efeito da familiaridade como o efeito do RVD variam ao longo da sessão. Uma análise de variância realizada com base na taxa de variação do desempenho através dos blocos mostra que o indice de discriminação melhora com uma taxa de $0,38 /$ bloco (EPM $=0,08$ ) no grupo que foi exposto aos estímulos repetidos, enquanto o desempenho do grupo exposto aos estímulos inéditos melhorou em uma taxa menor, de 0,15/ bloco $(E P M=0,08) F(1,30)=4,26, p=0,05, n_{p}{ }^{2}=0,12$ ).

Esses resultados evidenciam que a dica retroativa permite que a informação indicada seja recuperada de maneira mais eficiente, uma vez que o desempenho, tanto em termos de acurácia como em termos de TR, é significativamente melhor nas provas com dicas informativas que nas provas com dicas neutras. Esse efeito da dica independe da presença do RVD, dos blocos de provas nos dois de participantes, que trabalharam com estímulos Inéditos e Repetidos ao longo da sessão. Ou seja, o processo de recuperação baseado na dica retroativa não depende da familiaridade (repetição dos estímulos ao longo das provas b1, b2 e b3). 
O TR (Figura 2B) foi submetido à mesma analise de variância que o d'. De acordo com esta analise, o TRs é menor nas provas com dicas informativas $(T R=970 \mathrm{~ms} ; E P M=114 \mathrm{~ms})$ do que nas provas com dicas neutras ( $T R=1149 \mathrm{~ms} ; E P M=130 \mathrm{~ms}$ ) $\left[F(1,30)=26,52, p<0,001 ; n_{p}^{2}=0,47\right]$ e diminui em média 9oms do primeiro ao último bloco de provas $\left[F(2,60)=5,06 ; \mathrm{p}<0,001 ; n_{p}^{2}=0,33\right]$. Nenhum outro fator ou interação tem efeitos significativos sobre o TR.

\section{Discussão}

Neste estudo, investigamos o efeito da familiaridade com os estímulos sobre o processo de recuperação de representações visuais na memória de trabalho. A familiaridade foi manipulada entre dois grupos de participantes que foram expostos repetidamente a um mesmo conjunto de caracteres chineses (Grupo com Estímulos Repetidos), ou expostos a um novo conjunto de estímulos em cada prova (Grupo com Estímulos Inéditos). A suposição era que a apresentação repetida dos mesmos estímulos contribuiria para que os mesmos se tornassem mais familiares, levando a um processo de unitização de seus componentes, o que resultaria em uma representação que seria recuperada com mais eficiência do que estímulos inéditos e não unitizados. Além disso, consideramos que o processo de unitização tornaria a representação memorizada mais estável e dessa forma, menos sensivel à interferência de informações visuais irrelevantes, do que as representações não unitizadas.

Nossos resultados mostram que a vantagem proporcionada pela dica retroativa é significativa e, embora apresente uma tendência a aumentar através dos blocos da sessão, esse aumento não chega a ser significativo. De acordo com a nossa suposição inicial a vantagem proporcionada pela dica reflete um processo de recuperação ou de priorização da informação memorizada. Nossos resultados mostram que este processo de recuperação não varia de forma significativa ao longo da sessão, assim como não varia em função da familiaridade.

De maneira geral nossos resultados mostram que o desempenho melhora ao longo dos blocos de provas, mas essa melhora depende do tipo de estímulos. O desempenho não varia de forma significativa para o grupo que trabalhou com estímulos inéditos, mas melhora para o grupo que trabalhou com estímulos repetidos e mesmo para esse grupo, a melhora só acontece de forma significativa no terceiro bloco de provas. Ou seja, a exposição aos mesmos estímulos torna o seu processamento mais eficiente, mas isto só é evidente depois que se estabelece uma relação de familiaridade com os estímulos.

A melhora acentuada no desempenho ao longo dos blocos de provas do grupo que trabalhou com estímulos repedidos evidenciam que possivelmente existem diferenças importantes entre as características da representação visual para estímulos repetidos e para estímulos inéditos. Nossos dados corroboram a literatura, uma vez que reforçam a ideia que a exposição repetida dos mesmos estímulos gera novas associações (ou atualiza associações já existentes) de seus componentes, facilitando o seu reconhecimento (McClelland \& Rumelhart, 1985; Vagnot, 2014), melhorando tanto acurácia, tempo de resposta, quanto níveis de atividade cerebral quando esses estímulos são reapresentados em curtos intervalos de tempo (James \& Gauthier, 2006).

$O$ benefício gerado pela repetição dos estímulos é mais evidente no terceiro bloco ou etapa da sessão, quando podemos supor que o número de repetições dos itens já teria sido suficiente para que ocorresse o processo de familiarização. A familiaridade resulta da exposição repetida ao mesmo conjunto de estímulos, sendo considerada como uma forma indireta de avaliar a associação de seus componentes que ocorre na unitização (Perlman, Pothos, Edwards, \& Tzelgov, 2010). Da mesma forma, a literatura evidencia que a familiaridade permite a formação de uma representação mais unitizada para esses estímulos mais familiares (McClelland \& Rumelhart, 1985; Vagnot, 2014; James \& Gauthier, 2006; Kandel et al.; 2014; Delhaye \& Bastin, 2016).

Nós consideramos que a melhora do 
desempenho ao longo da sessão enfatiza a importância do treino/repetição para que a representação do estimulo seja de fato unitizada (McLauren \& Mackintosh, 2000; Schyns, Goldstone, \& Thibaut, 1998). Esse resultado aponta para a importância do desenvolvimento de unidades perceptivas (geradas a partir da exposição repetida aos estímulos) no processo de unitização dos itens memorizados (McLauren \& Mackintosh, 2000), sinalizando a necessidade de considerar o desempenho ao longo da interação com os estimulos como um fator importante no estudo do processo de unitização em futuros estudos experimentais. No entanto, ao contrário do que supúnhamos, o processo de unitização não foi acompanhado de uma diminuição do efeito do RVD. A hipótese levantada era de que o RVD teria um efeito prejudicial menos acentuado sobre o desempenho do grupo exposto aos estimulos repetidos, pois supúnhamos que esse grupo estaria trabalhando com representações mais familiares, mais unitizadas e, portanto, menos sensiveis à interferência visual irrelevante, o que não aconteceu. De fato, o efeito do RVD se mostrou mais acentuado exatamente sobre o desempenho do grupo que trabalhou com estimulos repetidos e não teve efeito algum sobre o desempenho do grupo que trabalhou com estímulos inéditos. Esse resultado, embora contrário à nossa suposição sugere que o resultado da exposição repetida ao mesmo conjunto de estímulos permitiu a codificação desses estímulos em termos visuais e que a recuperação dessas representações visuais tenha sido mais sensivel ao RVD (Valenti \& Galera, no prelo; Galera \& Quinn, 2014). Por outro lado, a ausência do efeito do RVD nas provas com estímulos inéditos sugere que as representações codificadas e recuperadas nessas provas tinham caracteristicas diferentes daquelas geradas a partir de estímulos repetidos. Também é possivel que o desempenho baixo obtido no grupo com estímulos inéditos tenha imposto um limite ao efeito do RVD. Esta questão, do efeito do RVD em tarefas de baixo desempenho, permanece em aberto. Essa questão é relevante para a compreensão do efeito do ruido em tarefas de reconhecimento e deve ser explorada em estudos futuros.

\section{Conclusão}

Em resumo, nossos resultados sugerem que a exposição repetida a um conjunto de estímulos possibilita a criação de representações mais unitizadas do que a de estímulos inéditos. Possivelmente as representações de estímulos repetidos são recuperadas para um estado ativo em termos de representações visuais sensiveis ao RVD, diferentemente das representações de estímulos inéditos. Embora a natureza da representação recuperada possa ser determinada pela familiaridade, essa não parece interferir no processo de recuperação da informação na memória de trabalho.

I.P e C.G planejaram, discutiram e escreveram o manuscrito.

\section{Referências}

Ahmad, F. N., Fernandes, M., Hockley, W. E. (2015). Improving associative memory in older adults with unitization. Neuropsychol. Dev. Cogn. Sect. B Aging Neuropsychol. Cogn. 22(4), 452-472. https://doi.org/1 $\underline{0.1080 / 13825585.2014 .980216}$

Baddeley, A. (2007). Working memory, thought, and action. Oxford: Clarendon.

Bader, R., Opitz, B., Reith, W., Mecklinger, A. (2014). Is a novel conceptual unit more than the sum of its parts?: FMRI evidence from an associative recognition memory study. Neuropsychologia, 61 . 123-134. https://doi.org/10.1016/j.neuropsychologia.2014.06.006

Bower, G. H. (2000). A brief history of memory research. The Oxford handbook of memory, 3-32.

Brady, T. F., Störmer, V. S., \& Alvarez, G. A. (2016). Working memory is not fixed-capacity: More active storage capacity for real-world objects than for simple stimuli. Proceedings of the National Academy of Sciences, 113(27), 7459-7464. https://doi.org/10.1073/ pnas. 1520027113

D'Angelo, M. C., Smith, V. M., Kacollja, A., Zhang, F., Binns, M. A., Barense, M. D., \& Ryan, J. D. (2016). The effectiveness of unitization in mitigating age-related relational learning impairments depends on existing cognitive status. Aging, Neuropsychology, and Cognition, 23(6), 667-690. https://doi.org/10.1080/138255 $\underline{85.2016 .1158235}$

Delhaye, E., \& Bastin, C. (2018). The impact of aging on associative memory for preexisting unitized associations. Aging, Neuropsychology, and Cognition, 25(1), 70-98. https://doi.org/10.1080/13825585.2016 .1263725 
Galera, C., \& Quinn, G. (2014, julho). Visual buffer and retrieval of visual information from working memory. Comunicação apresentada em International Conference on Working Memory. Cambridge, UK.

Goldstone, R. L. (2000). Unitization during category learning. Journal of Experimental psychology. Human Perception and Performance, 26, 86-112. https:// doi.org/10.1037/0096-1523.26.1.86

Griffin, I. C., \& Nobre, A. C. (2003). Orienting attention to locations in internal representations. Journal of Cognitive Neuroscience, 15, 1176-1194. https://doi. org/10.1162/089892903322598139

Hebb, D. O. (1961). Distinctive features of learning in the higher animal. In B. F. Delafresnaye (Ed.), Brain mechanisms and learning (pp. 37-46). Oxford: Blackwell.

James, T. W., \& Gauthier, I. (2006). Repetition induced changes in BOLD response reflect accumulation of neural activity. Human brain mapping, 27(1), 37-46. https://doi.org/10.1002/hbm.20165

Kandel, E., Schwartz, J., Jessell, T., Siegelbaum, S., \& Hudspeth, A. J. (2014). Principios de Neurociências-5. AMGH Editora.

Kibbe, M. M., \& Feigenson, L. (2016). Infants use temporal regularities to chunk objects in memory. Cognition, 146, 251-263. https://doi.org/10.1016/j. cognition.2015.09.022

Kosslyn, S. M. Image and Brain: The Resolution of the Imagery Debate. Cambridge: Mit Press, p 516, 1994.

Kosslyn, S. M., Thompson, W. L., \& Ganis, G. (2006). The case for mental imagery. Oxford University Press.

Landman, R., Spekreijse, H., \& Lamme, V. A. F. (2003). Large capacity storage of integrated objects before change blindness. Vision Research, 43, 149-164. https://doi.org/10.1016/s0042-6989(02)00402-9

Lepsien, J., \& Nobre, A. (2006). Cognitive control of attention in the human brain: Insights from orienting attention to mental representations. Brain Research, 1105. 20-31. http://dx.doi.org/10.1016/j.brainres.2006.03.033

Li, B., Mao, X., Wang, Y., \& Guo, C. (2017). Electrophysiological Correlates of Familiarity and Recollection in Associative Recognition: Contributions of Perceptual and Conceptual Processing to Unitization. Front. Hum. Neurosci. 11:125. http://dx.doi.org/10.3389/ fnhum.2017.00125

McClelland, J. L., \& Rumelhart, D. E. (1985). Distributed memory and the representation of general and specific information. Journal of Experimental Psychology: General, 114, 159-188. http://dx.doi. org/10.1037/oog6-3445.114.2.159

McLaren, I. P. L., \& Mackintosh, N. J. (2000). An elemental model of associative learning: I. Latent inhibition and perceptual learning. Animal Learning \& Behavior, 28, 211-246. http://dx.doi.org/10.3758/BF03200258
Memel, M., \& Ryan, L. (2017). Visual integration enhances associative memory equally for young and older adults without reducing hippocampal encoding activation. Neuropsychologia, 100, 195-206. https://doi.org/10.1016/i.neuropsychologia.2017.04.031

Miller, G. A. (1956). The magical number seven, plus or minus two: some limits on our capacity for processing information. Psychological Review, 63(2), 81. http://dx.doi.org/10.1037/ho043158

Orme, E., Brown, L., \& Riby, L. (2017). Retrieval and monitoring processes during visual working memory: An ERP study of the benefit of visual semantics. Frontiers in Psychology, 8, 1080. http://doi.org/10.3389/ fpsyg.2017.01080

Parks, C. M., \& Yonelinas, A. P. (2015). The importance of unitization for familiarity-based learning. Journal of Experimental Psychology: Learning, Memory, and Cognition, 41(3), 881-903. http://dx.doi.org/10.1037/ xlmo000068

Perlman, A., Pothos, E. M., Edwards, D. J., \& Tzelgov, J. (2010). Task-relevant chunking in sequence learning Journal of Experimental Psychology: Human Perception and Performance, 36(3), 649-661. http://dx.doi. org/10.1037/a0017178

Pucci, I. W; Galera, C. A (2014, setembro). Recuperação da Informação da memória de Trabalho Visual: A necessidade de um buffer visual. Pôster apresentado no $22^{\circ}$ Simpósio Internacional de Iniciação Científica da USP (SIICUSP), Ribeirão Preto, SP. https://doi. org/10.11606/d.59.2016.tde-05052016-182541

Quinn, J. G., \& McConnell, J. (1996). Indications of the functional distinction between the components of visual working memory. Psychologische Beitrage, 38(3-4), 355-367.

Reder, L. M., Liu, X. L., Keinath, A., \& Popov, V. (2016) Building knowledge requires bricks, not sand: The critical role of familiar constituents in learning. Psychonomic Bulletin \& Review, 23, 271-277. http://dx. doi. org/10.3758/s13423-015-0889-1

Reder, L. M., Paynter, C., Diana, R. A., Ngiam, J., \& Dickison, D. (2007). Em B. Ross\& A. S. Benjamin (Eds.). The psychology of learning and motivation (pp. 271-312). New York, NY: Academic Press.

Riby, L. M., \& Orme, E. (2013). A familiar pattern? Semantic memory contributes to the enhancement of visuo-spatial memories. Brain and cognition, 81(2), 215-222. http://dx.doi.org/10.1016/j.bandc.2012.10.011

Robey, A., \& Riggins, T. (2017). Increasing relational memory in childhood with unitization strategies. Memory \& Cognition, 46(1), 100-111.

Schneider, W., Eschman, A., \& Zuccolotto, A. (2002). E-Prime reference Guide (Version 1.2). Psychology Software Tools Inc.

Schyns, P. G., Goldstone, R. L., \& Thibaut, J. P. (1998). The development of features in object concepts. The Behavioral and Brain Sciences, 21, 1-17. https://doi. org/10.1017/s0140525 $\times 98000107$ 
Shiffrin, P. G., \& Lightfoot, N. (1997). Perceptual learning of alphanumeric-like characters. Em R. L. Goldstone, P. G. Schyns, \& D. L. Medin (Eds.). The psychology of learning and motivation (Vol. 36, pp. 45-82). San Diego, CA: Academic Press. https://doi.org/10.1016/ s0079-7421(08)60281-9

Snodgrass, J. G., \& Corwin, J. (1988). Pragmatics of measuring recognition memory: applications to dementia and amnesia. Journal of Experimental Psychology: General, 117(1), 34-50. https://doi. org/10.1037/0096-3445.117.1.34

Souza, A. S., \& Oberauer, K. (2016). In search of the focus of attention in working memory: 13 years of the retro-cue effect. Attention, Perception, \& Psychophysics, 78(7), 1839-1860. http://dx.doi.org/10.3758/ s13414-016-1108-5

Vagnot, C. (2014). Efficacité d'une activité discriminante: quand isolation et action participent à l'émergence d'un jugement de reconnaissance. Dissertação de doutorado, Psychologie Université Paul Valéry-Montpellier-III, França.

Valenti, L., \& Galera, C. (no prelo). Dynamic Visual Noise has the same effect on Visual Memory and Visual Imagery tasks. Psychology \& Neuroscience. https://doi.org/10.1037/pneo000183

\section{Endereço para correspondência:}

César Galera (Av. Bandeirantes, n’ 3900, Ribeirão Preto, São Paulo, CEP: 14040-901; Tel: (16)33153760 / (16) 997343320; algalera@usp.br).

\section{Autor I}

Nome: Isabella Wada e Pucci

Titulação Acadêmica: Bacharel em Psicologia.

Afiliação Institucional: Universidade de São Paulo (USP-Ribeirão Preto)

\section{Autor II \\ Nome: César Galera}

Titulação Acadêmica: Doutor.

Afiliação Institucional: Universidade de São Paulo (USP-Ribeirão Preto) 